\title{
Elaboración y valoración del hierro en el pan enriquecido con harina de quinua (Chenopodium quinoa w.) y soja (Glycine max)
}

\author{
Diana Gloria Mendoza-Pérez ${ }^{1 *}$ y Félix Nicolás Palacios-Morales ${ }^{1}$ \\ ${ }^{1}$ E.A.P Nutrición Humana, Facultad de Ciencias de la Salud, Universidad Peruana Unión, EAP. Nutrición Humana Alt. Km.19, \\ Carretera Central, Ñaña, Chosica, Lima
}

\begin{abstract}
RESUMEN
Objetivo: Elaborar y valorar el hierro en el pan enriquecido con harina de quinua (Chenopodium quinoa W.) y soja (Glycine max); para este fin se enriqueció el pan, planteando dos formulaciones. Materiales y métodos: Se evaluó la cantidad de hierro en la harina de quinua y soja, prueba de aceptabilidad y la valoración de la cantidad de hierro en el producto final, se realizó también a los panes elaborados el análisis proximal, en el Laboratorio Calidad Total de la Universidad Nacional Agraria de la Molina (UNALM). Resultados: Los resultados de hierro en el producto final fueron para la primera formulación $6.2 \mathrm{mg} / 100 \mathrm{~g}$, segunda formulación $6.0 \mathrm{mg} / 100 \mathrm{~g}$ de producto, mostró diferencia significativa con el contenido de hierro con el pan francés popular. En la prueba de aceptabilidad se apreció mayor aceptación para la primera formulación. Conclusiones: El pan enriquecido con harina de quinua y soja no solo contiene cantidades superiores de hierro frente al pan francés, también en proteínas con contenido de aminoácidos esenciales; haciendo de este un pan con alto valor nutritivo.
\end{abstract}

Palabras clave: Quinua, soja, miligramos, hierro, formulación

\begin{abstract}
Objetive: To develop and evaluate the iron in enriched bread flour quinoa (Chenopodium quinoa W.) and soybeans (Glycine max), for this purpose, enriched bread, raising two formulations. Materials and Methods: We evaluated the amount of iron in the quinoa and soy flour, acceptability test and evaluation of the amount of iron in the final product was also breads made proximal analysis, Total Quality in the Laboratory National Agrarian University la Molina (UNALM). Results: The results of iron in the final product for the first formulation were $6.2 \mathrm{mg} / 100 \mathrm{~g}$, second formulation $6.0 \mathrm{mg} / 100 \mathrm{~g}$, showed a significant difference in iron content with popular French bread. In the test of acceptability presented greater acceptance for the first formulation. Conclusions: The bread enriched with quinoa and soy flour not only contains higher amounts of iron against the French bread, also in proteins containing essential amino acids, making this a highly nutritious bread.
\end{abstract}

Keywords: Quinoa, soy, milligrams iron formulation 


\section{INTRODUCCIÓN}

En el Perú los productos obtenidos de las industrias de panificación tienen gran aceptación por los sectores rurales y urbanos; como es el caso del pan, alimento que tiene gran importancia en todos los niveles sociales de la población.

Desde mediados del siglo pasado las grandes, medianas y pequeñas industrias panificadoras han utilizado la harina de trigo, como la principal materia prima para la elaboración del pan, constituyéndose a través del tiempo en el elemento más importante para su producción ${ }^{1}$. Ya que de su calidad depende no solo de las características del producto final, sino también del aporte nutricional, mientras tanto, estadísticas de la Organización Mundial de la Salud (OMS) ${ }^{2}$ indican que el $80 \%$ de la población mundial, es decir que más de los 2/3 (de 4 mil millones de personas), recurren al pan como dieta diaria básica.

Este producto, teniendo tal aceptabilidad en el mercado, no provee al consumidor elementos nutritivos que vayan a satisfacer las necesidades nutricionales, siendo la anemia una problemática en nuestro país; la elaboración del pan enriquecido con quinua y soya, conteniendo ambos fuente natural de hierro, se presenta como una alternativa nutricional válida.

De esta manera se pretende fomentar la producción de quinua y soja en el país, potenciar las características nutritivas $^{3}$, aprovechar sus bondades intrínsecas como es la proteína, grasas insaturadas, vitaminas, minerales, carbohidratos y el alto contenido de fibra dietética, cuyos componentes se concentran en el alimento básico de la humanidad, como es el pan ${ }^{4}$.

El presente trabajo tuvo como objetivo: Elaborar y valorar el contenido de hierro en el pan enriquecido con harina de quinua (Chenopodium quinoa $W$.) y harina de soja (Glycine max).

\section{MATERIAL Y MÉTODOS}

El estudio fue de tipo tecnológico, ya que se desarrolló un nuevo producto. En cuanto al enfoque cuantitativo corresponde al tipo preexperimental, porque el instrumento se aplicó solo a un grupo de la población. El trabajo de investigación se llevó a cabo en las instalaciones del Laboratorio de Panificación de la UNALM (Universidad Nacional Agraria la Molina).

Se optimizó el uso de quinua blanca, variedad más consumida por la población y accesible su compra en la provincia de Huancayo, distrito de Pampas; y la soja de la provincia de Chanchamayo, provenientes del departamento de Junín.
Para eliminar la presencia de saponina se realizó el lavado de los granos de quinua por cuatro veces continuas hasta obtener una muestra sin espuma. Posteriormente se realizó el proceso de secado por un tiempo de 3: 30 horas. Luego se realizó la molienda de quinua y soja con molino de martillo marca PULLMAN de la Molinera El Progreso S.A.C y envasadas en bolsas de polietileno en la ciudad de Huancayo y la harina de trigo panadera marca ANITA fue adquirida en la Panadería Sol de Vitarte.

Para evaluar el contenido de hierro en la harina de quinua y soja, fueron pesadas y envasadas conteniendo cada una $400 \mathrm{~g}$ de cada muestra, para el análisis fueron llevados al Laboratorio Calidad Total de la Universidad Nacional Agraria de la Molina (UNALM).

\section{Insumos}

- Harina de trigo panadera

- Harina de quinua

- Harina de soja

- Levadura fresca prensada

- Sal industrial

- Grasa vegetal

- Agua

- Azúcar rubia

\section{Materiales y equipos}

- Utensilios para panificación

- Lapiceros

- Cuaderno de notas

- Software EXCEL

- Amasadora sobadora: Ficha técnica

- Divisora de masa: Ficha técnica

- Cámara de fermentación: Ficha técnica

- Horno rotativo: Ficha técnica

- Balanza digital: Ficha técnica

- Mesa para moldeo: Ficha técnica

\section{Formulación para la elaboración del pan}

El proceso de elaboración de pan enriquecido con harina de quinua y soja es conforme a las fórmulas que a continuación se detallan:

Formulación $1 \longrightarrow 70 \%$ harina de trigo, $10 \%$ harina de quinua, $20 \%$ harina de soja

Formulación $2 \longrightarrow 70 \%$ harina de trigo, $15 \%$ harina de quinua, $15 \%$ harina de soja.

\section{Métodos de Análisis}

Métodos de análisis físico-químico

En la harina de quinua, harina de soja se realizó:

Análisis proximal de hierro: Método AOAC 975.03 Ca 3 Ed.18 Pág. 32005. 


\section{Análisis del producto final}

Análisis físico

Peso: Se realizó después de elaborado del pan, por pesado directo en una balanza de precisión con una resolución de $0,1 \mathrm{k}$.

Análisis proximal químico

- Humedad: Método NTP 206.011 2011.

- Cenizas: Método AOAC 930.22 Ca 32 Ed. 18 Pág. 572011.

- Energía Total: Método por cálculo MS-INN Collazos 1993.

- Proteína: Método AOAC 935.39 Ca 32 Ed. 18 Pág. 592011.

- Grasa: Método AOAC 935.39 Ca 32 Ed. 18 Pág. 592011.

- Carbohidratos: Método por diferencia MSINN Collazos 1993.

- Hierro: Método AOAC 985.35 Ca 50 Ed. 18 Pág. 15 -17 2011.

\section{Prueba de aceptabilidad}

\section{Prueba hedónica}

Las pruebas hedónicas están destinadas a medir cuánto agrada o desagrada un producto ${ }^{4}$. Para estas pruebas se utilizan preferencias categorizadas, que pueden tener diferente número de categorías y que comúnmente van desde "me gusta mucho", "me gusta poco", "me es indiferente", "me disgusta un poco", "me disgusta mucho". La población elegida para la evaluación debe corresponder a los consumidores potenciales o habituales del producto en estudio.

Es una prueba sencilla de aplicar y no requiere entrenamiento o experiencia por parte de los jueces consumidores.

\section{Aplicación de la prueba}

Para determinar el nivel de agrado de los panes, se les sometió a una prueba de aceptabilidad (prueba hedónica) cuya escala estructurada fue de 5 puntos y siendo las alternativas de respuesta las siguientes: "Me gusta mucho" (5 puntos) "me gusta poco" (4 puntos) "me es indiferente" (3 puntos) "me disgusta un poco" (2 puntos) "me disgusta mucho" (1 punto). Para la prueba se contó con el apoyo de 30 jueces no entrenados pertenecientes a nivel universitario como se indica a continuación:

30 estudiantes universitarios pertenecientes a la UNALM y cuyas edades oscilaron entre 19 y 30 años.

La prueba se realizó entre las 11 a $12 \mathrm{hrs}$. y entre las 16 y $17 \mathrm{hs}$. las muestras se sirvieron entre $18^{\circ}$ y $22^{\circ} \mathrm{C}$, temperatura a la cual se consumen normalmente el producto.

Los panes fueron cortados adecuadamente y pesada cada muestra $(8 \mathrm{~g})$ para la degustación de los jueces.

\section{RESULTADOS Y DISCUSIÓN}

La tabla 1 muestra los resultados del análisis físico-químico en el que se determina el contenido de hierro en la harina de quinua y soja, expresados como $\mathrm{mg} / 100 \mathrm{~g}$. La harina de quinua presenta una cantidad de hierro de $7.7 \mathrm{mg} / 100 \mathrm{~g}$ de muestra muy por encima del valor mostrado en la Tabla de Composición de Alimentos ${ }^{5}$ que es de solo $3.7 \mathrm{mg} / 100 \mathrm{~g}$.

En los respecta a la muestra de harina de soja presenta una cantidad de hierro de $9.6 \mathrm{mg} / 100 \mathrm{~g}$, resultado que no se puede comparar con el de la Tabla de Composición de Alimentos ${ }^{5}$, ya que esta no contiene la información de la harina de esta leguminosa; pero sí del grano de soja que contiene $8.3 \mathrm{mg} / 100 \mathrm{~g}$.

Tabla 1

Análisis físico - químico de la harina de quinua y soja

\begin{tabular}{|c|c|c|}
\hline Tipo de análisis & Harina de quinua & Harina de soja \\
\hline Hierro $(\mathrm{mg} / 100 \mathrm{~g}$ de muestra $)$ & 7.7 & 9.6 \\
\hline
\end{tabular}

El análisis proximal energía total, proteína, grasa, carbohidratos y hierro de ambas muestras enriquecidas con harina de quinua y soja, se muestra en la Tabla 2, en la cual no se observó diferencia significativa en la cantidad de hierro. En Perú León y Villacorta ${ }^{6}$ elaboraron un pan con sustitución parcial de harina de trigo por arracacha que presentó proteína $8,32 \%$; grasa $10,11 \%$ y carbohidratos $55,13 \%$ con un valor energético de
$344,79 \mathrm{Kcal} / 100 \mathrm{~g}$; hierro (>5 mg/100g). En Ecuador Ordóñez y Oviedo $^{7}$ desarrollaron una propuesta distinta y original para el proceso de elaboración de un pan que permitió utilizar materias primas que reemplazaron parcialmente a la harina de trigo como elemento tradicional para su elaboración, con harina de centeno, maíz; presentó energía 339,49 kcal/100g; Carbohidratos 58,14 g/100 g; Proteínas $14.61 \mathrm{~g} / 100 \mathrm{~g}$; Hierro 5,03 mg/100g. 
Tabla 2

Análisis físico-químico de los panes enriquecidos con harina de quinua y soja

\begin{tabular}{lcc}
\hline \multicolumn{1}{c}{ Tipo de análisis } & $110(*)$ & $111(*)$ \\
\hline Energía total (Kcal/100 g de muestra original) & 295.6 & 310.6 \\
Carbohidratos (g/100 g de muestra original & 52.5 & 53.9 \\
Grasa (g/100 g de muestra original) & 4.0 & 5.0 \\
Proteína (g/100 g de muestra original) & 12.4 & 12.5 \\
Humedad (g/100 g de muestra original) & 29.0 & 26.5 \\
Ceniza (g/100 g de muestra original) & 2.1 & 2.1 \\
Hierro (g/100 g de muestra original) & 6.2 & 6.0 \\
\hline
\end{tabular}

(*) $70 \%$ harina de trigo, 10\% harina de quinua, 20\% harina de soja (según la formulación)

(**) $70 \%$ harina de trigo, 15\% harina de quinua, 15\% harina de soja (según la formulación)

Tabla 3

Contenido de hierro en el pan enriquecido con harina de quinua y soja F1 (*), F2 (**), pan francés y pan francés fortificado con hierro

\begin{tabular}{|c|c|c|c|c|}
\hline Tipo de análisis & $\begin{array}{l}\text { Pan enriquecido con } \\
\text { harina de quinua y soja } \\
\text { F1(*) }\end{array}$ & $\begin{array}{l}\text { Pan enriquecido con } \\
\text { harina de quinua y soja } \\
\text { F2(**) }\end{array}$ & Pan francés & $\begin{array}{c}\text { Pan francés } \\
\text { fortificado con } \\
\text { hierro }\end{array}$ \\
\hline $\begin{array}{c}\text { Hierro }(\mathrm{mg} / 100 \mathrm{~g} \\
\text { de muestra) }\end{array}$ & 6.2 & 6.0 & 1.0 & 3.14 \\
\hline
\end{tabular}

(*) $70 \%$ harina de trigo, $10 \%$ harina de quinua, 20\% harina de soja (según la formulación)

(**) $70 \%$ harina de trigo, $15 \%$ harina de quinua, 15\% harina de soja (según la formulación)

En la tabla 3 se muestra los resultados de hierro del producto final de ambas muestras enriquecidas con harina de quinua, soja e información del análisis del pan francés según la Tabla de Composición de Alimentos $^{5}$ en $1 \mathrm{mg} / 100 \mathrm{~g}$ mostrando una diferencia significativa en el contenido de hierro.

Tabla 4

Grado de aceptabilidad de los panes en los estudiantes universitarios

\begin{tabular}{lcccc}
\hline \multirow{2}{*}{ Grado de aceptabilidad } & \multicolumn{2}{c}{ Muestra 1 $(*)$} & \multicolumn{2}{c}{ Muestra 2(**) } \\
& N. & $\%$ & N. & $\%$ \\
\hline Me gusta mucho & 18 & 60 & 3 & 10 \\
Me gusta poco & 10 & 33.3 & 18 & 60 \\
Me es indiferente & 2 & 6.7 & 9 & 30 \\
Me disgusta un poco & 0 & 0 & 0 & 0 \\
Me disgusta mucho & 0 & 0 & 0 & 0 \\
\hline
\end{tabular}

(*) $70 \%$ harina de trigo, $10 \%$ harina de quinua, 20\% harina de soja (según la formulación)

(**) $70 \%$ harina de trigo, $15 \%$ harina de quinua, 15\% harina de soja (según la formulación)

Los panes enriquecidos que fueron sometidos a la prueba de aceptabilidad, en la tabla 4 se muestran las alternativas de respuesta con el número de estudiantes que respondieron ante tales alternativas y el porcentaje que representa del total de ellos. 
Se aprecia claramente que el $60 \%$, en la muestra 1 , calificaron al pan enriquecido dentro de la escala "me gusta mucho". Para la muestra 2, el 10\% calificaron dentro de la escala "me gusta mucho". Los panes

Tabla 5

Análisis de varianza de prueba de aceptabilidad enriquecidos obtuvieron puntajes por encima de los 4 puntos, lo que nos indica que se encuentra dentro del rango "me gusta" y "me gusta mucho".

\begin{tabular}{ccccc}
\hline Fuente de variación & $\begin{array}{c}\text { Suma de } \\
\text { cuadrados }\end{array}$ & $\begin{array}{c}\text { Grados de } \\
\text { Libertad }\end{array}$ & $\begin{array}{c}\text { Cuadrados } \\
\text { medios }\end{array}$ & $\begin{array}{c}\text { Razón F } \\
\text { calculada }\end{array}$ \\
\hline Tratamiento & 8.07 & 1 & 8.07 & 39.43 \\
Jueces & 16.33 & 29 & 0.56 & 2.75 \\
Error & 5.93 & 29 & 0.20 & \\
\hline
\end{tabular}

\begin{tabular}{|c|c|c|}
\hline Valor critico & $\sqrt{\frac{2}{r}(C M E) F_{1-\alpha, 1, n-k}}$ & 0.23 \\
\hline $\begin{array}{c}\text { Diferencia de } \\
\text { medias }\end{array}$ & $\mathrm{X}_{1}-\mathrm{X}_{2}$ & 0.73 \\
\hline
\end{tabular}

Se observa, en la tabla 5, que el valor F calculado para tratamientos es de 39,42 y es superior al valor $F$ tabulado que es de 4,18, se llega a la conclusión de que existe una diferencia significativa $\mathrm{p}=0,05$, entre los puntajes hedónicos promedio, para las dos tratamientos de pan. El valor F calculado para los jueces fue de 2,75.

Este valor fue mayor al valor $\mathrm{F}$ tabulado que es de 1,86 , por lo que también se encontró un efecto

Tabla 6

Prueba de muestras independientes significativo de los jueces.

El análisis de varianza (ANOVA) indicó que había diferencias significativas entre los dos tratamientos de pan. Al igual se muestra que para determinar qué tratamiento de pan diferían significativamente la una de la otra, se utilizó la prueba de comparación múltiple, donde la diferencia entre los pares de medias resulta ser superior al valor crítico, por lo tanto, es significativo.

\begin{tabular}{|c|c|c|c|c|c|c|c|c|c|}
\hline & $\mathrm{F}$ & Sig. & $\mathrm{T}$ & Gl & $\begin{array}{c}\text { Sig. } \\
\text { (bilateral) }\end{array}$ & $\begin{array}{l}\text { Diferencia } \\
\text { de medias }\end{array}$ & $\begin{array}{c}\text { Error tí de la } \\
\text { diferencia }\end{array}$ & $\begin{array}{r}95 \% \text { Inte } \\
\text { confianz } \\
\text { difere }\end{array}$ & $\begin{array}{l}\text { valo de } \\
\text { para la } \\
\text { cia }\end{array}$ \\
\hline $\begin{array}{l}\text { Aceptación del } \\
\text { Producto }\end{array}$ & Inferior & Superior & Inferior & Superior & Inferior & Superior & Inferior & Superior & Inferior \\
\hline $\begin{array}{l}\text { No se han asumido } \\
\text { varianzas iguales }\end{array}$ & & & 4.167 & 57.948 & .000 & .667 & .160 & .346 & .987 \\
\hline
\end{tabular}

En la tabla 6 se observa, en cuanto a la prueba de Levene para contrastar hipótesis de igualdad de varianzas, que no se rechaza la hipótesis de igualdad de varianzas, pues el valor $p$ es 0.149 mayor a $\alpha=0.05$. Luego, para contrastar la diferencia de medias se eligió la alternativa de igualdad de varianzas, cuyo valor $\mathrm{t}=$ 4.167 tiene una probabilidad asociada (valor p, sig.
Bilateral) de 0.000 la cual, al ser menor que $\alpha=0.05$, sugiere rechazar la hipótesis nula, ya que las medias poblacionales son iguales; por lo tanto, se concluye que existen diferencias significativas respecto a la aceptación de ambos panes entre la formulación 1 y la formulación 2 . 
Tabla 7

Estadísticos de grupo

\begin{tabular}{llccc}
\hline & Grado de aceptación de producto & N. ${ }^{\circ}$ & Media & $\begin{array}{c}\text { Eesviación tí } \\
\text { media }\end{array}$ \\
\hline $\begin{array}{c}\text { Aceptación del } \\
\text { producto }\end{array}$ & Aceptación de la formulación 1 & 30 & 4.47 & .629 \\
& Aceptación de la formulación 2 & 30 & 3.80 & .610 \\
\hline
\end{tabular}

En la tabla 7 se observa que la formulación 1 presenta más grado de aceptabilidad que la formulación 2 .

La incorporación de harina de quinua y soja en la fórmula para elaborar el pan enriquecido tipo francés es una buena alternativa para proporcionar hierro "no hemo" a grupos vulnerables de la población. Para el presente estudio, se eligió el pan por ser un producto que es consumido masivamente por la población. El hierro no hemo presente en este pan, mencionado anteriormente, requiere de un potenciador para su absorción, como la vitamina $\mathrm{C}$ y el ácido málico, presentes en las manzanas y el tartárico en el jugo de uvas. Por lo cual se recomienda el consumo de este pan enriquecido, acompañado de los potenciadores para su mejor absorción. Las proporciones de harina de quinua y soja fueron establecidas con la finalidad de que el pan no cambie sus características gustativas y organolépticas, donde la cantidad de hierro por unidad de pan aportase a la dieta un porcentaje significativo de los requerimientos promedio.

La muestra 1 obtuvo gran aceptabilidad, cuyo aporte es de $6.2 \mathrm{mg} / 100 \mathrm{~g}$ de hierro, por cada $100 \mathrm{~g}$ de muestra, lo que significaría que cubre el $88,6 \%$ del requerimiento en niños de 1 a 3 años (ambos sexos), 62\% de 4 a 8 años (ambos sexos); 77,5\% en hombres de 9 a 15 años; $56.4 \%$ de 14 a 18 años; $77,5 \%$ de 19 a $>70$ años; $77,5 \%$ en mujeres de 9 a 13 años; 56,4\% de 14 a 18 años; $41,3 \%$ de 19 a 50 años; $77,5 \%$ en 50 a > 70 años; en gestantes 20,7\%;detallada en la Tabla 1. Diseñada en base a los estándares del requerimiento diario de ingesta de hierro de la información arriba descritos en hombres y mujeres desde un año, gestantes y mayores de 70 años, han servido para establecer los datos porcentuales que han sido adecuados según el contenido de hierro de este pan enriquecido, según el RDI para este estudio en las diferentes etapas de la vida.

Tabla 8 .

Porcentaje de adecuación de hierro relacionado con el RDI en las diferentes etapas de la vida

\begin{tabular}{ccc}
\hline & $\begin{array}{c}\text { RDI } \\
(\mathrm{mg} / \mathrm{d})\end{array}$ & $\begin{array}{c}\text { Porcentaje (\%) de adecuación, según contenido } \\
\text { de hierro del pan enriquecido }(*)\end{array}$ \\
\hline Niños 1 a 3 años (ambos sexos) & 7 & 88,6 \\
4 a 8 años (ambos sexos) & 10 & 62 \\
Hombres & & 58,14 \\
9 a 15 años & 8 & 77,5 \\
14 a 18 años & 11 & 56.4 \\
19 a $>70$ años & 8 & 77,5 \\
Mujeres & & \\
9 a 13 años & 8 & 77,5 \\
14 a 18 & 15 & 56.4 \\
19 a 50 años & 18 & 41,3 \\
50 a $>70$ años & 8 & 77,5 \\
Gestantes & 30 & 20.7 \\
\hline
\end{tabular}

Fuente: Elaboración en la investigación "Elaboración y valoración del hierro en el pan enriquecido con harina de quinua (Chenopodium quinoa w.) y soja (Glycine max)

(*) $70 \%$ harina de trigo, 10\% harina de quinua, 20\% harina de soja (según la formulación)

Food and Agriculture Organization/World Health Organization

(FAO/WHO) Requirements of vitamin A, iron, folate and vitamin B12; 2002. 
El aporte de hierro en una unidad de pan francés popular de $30 \mathrm{~g}$ es de $0.3 \mathrm{mg}$, y en el pan enriquecido con harina de quinua y soja de $30 \mathrm{~g}$ es de $1.86 \mathrm{mg}$ de hierro; siendo este un aporte importante, se muestra como buena alternativa para el consumo diario, aprovechando que el pan tiene un lugar prioritario en la mesa de todo nivel socioeconómico.

Tabla 9.

Costos de producción del pan enriquecido a nivel experimental en Laboratorio (Tratamiento T1)

\begin{tabular}{|c|c|c|}
\hline $\begin{array}{l}\text { Materia Prima } \\
\text { Insumos }\end{array}$ & Unidad & Precio (Soles) \\
\hline Harina de trigo & $0.700 \mathrm{~g}$. & 1.68 \\
\hline Harina de soja & $0.200 \mathrm{~g}$. & 1.20 \\
\hline Harina de quinua & $0.100 \mathrm{~g}$. & 0.90 \\
\hline Sal & $0.020 \mathrm{~g}$. & 0.20 \\
\hline Azúcar rubia & $0.060 \mathrm{~g}$. & 0.14 \\
\hline LFP (Levadura fresca prensada) & $0.030 \mathrm{~g}$. & 0.45 \\
\hline Grasa vegetal & $0.050 \mathrm{~g}$. & 0.30 \\
\hline Agua potable & $0.470 \mathrm{~g}$. & 1.00 \\
\hline Costos directos & & 0.80 \\
\hline Costos indirectos & & 0.50 \\
\hline Costo total & & 7.17 \\
\hline
\end{tabular}

Fuente: Elaboración en la investigación "Elaboración y valoración del Hierro en el pan enriquecido con harina de quinua (Chenopodium quinoa w.) soja (Glycine max).

En el Perú se elaboró un pan con sustitución parcial de harina de trigo por arracacha presentando contenidos de proteína $8,32 \%$; grasa $10,11 \%$ y carbohidratos $55,13 \%$ con un valor energético de 344,79 kcal/100 g; hierro (> $5 \mathrm{mg} / 100 \mathrm{~g}) 6$. En el Ecuador se desarrolló una propuesta distinta y original para el proceso de elaboración de un pan que permitió utilizar materias primas que reemplazaron parcialmente a la harina de trigo como elemento tradicional para su elaboración, con harina de centeno, maíz; presentando energía 339,49 $\mathrm{kcal} / 100 \mathrm{~g}$; Carbohidratos 58,14 g/100 g; proteínas 14.61 $\mathrm{g} / 100 \mathrm{~g}$; hierro $5,03 \mathrm{mg} / 100 \mathrm{~g} 7$. La alternativa que se presenta, en este trabajo de investigación, muestra que los panes enriquecidos con harina de quinua y soja, en cuanto al contenido de hierro es de $6.2 \mathrm{mg} / 100 \mathrm{~g}$ y $6.0 \mathrm{mg} / 100 \mathrm{~g}$ respectivamente, siendo mayor, frente a los resultados de la investigaciones mencionadas anteriormente. Para determinar los costos de producción del pan enriquecido, se toma en cuenta el total del costo experimental del primer tratamiento, en la tabla 9, se muestra los costos de las materias primas e insumos empleados en el proceso.

Tabla 10.

Costo de producción de pan francés popular

\begin{tabular}{lcc}
\hline \multicolumn{1}{c}{$\begin{array}{c}\text { Materia Prima } \\
\text { Insumos }\end{array}$} & Unidad & Precio (Soles) \\
\hline Harina de trigo & $0.1000 \mathrm{~g}$. & 2.50 \\
Sal & $0.020 \mathrm{~g}$. & 0.20 \\
Azúcar rubia & $0.060 \mathrm{~g}$. & 0.14 \\
LFP (Levadura fresca prensada) & $0.035 \mathrm{~g}$. & 0.53 \\
Grasa vegetal & $0.050 \mathrm{~g}$. & 0.30 \\
Agua potable & $0.500 \mathrm{~g}$. & 1.06 \\
Costos directos & & 0.80 \\
Costos indirectos & & 0.50 \\
Costo total & & $\mathbf{6 . 0 3}$ \\
\hline
\end{tabular}

Fuente: Elaboración en la investigación "Elaboración y valoración del hierro en el pan enriquecido con harina de quinua (Chenopodium quinoa w.) y soja (Glycine max)

En consecuencia, como resultado se tiene que para el primer tratamiento se obtuvo 33 panes, los cuales tendrán un costo de 0.13 céntimos por cada unidad de pan enriquecido aproximadamente de $30 \mathrm{~g}$; se toma referencia en la tabla 10 la elaboración del pan francés popular a un costo de 0.10 céntimos por cada unidad de pan de $30 \mathrm{~g}$. 
Tabla 11.

Composición química del pan enriquecido con harina de quinua y soja y pan francés

\begin{tabular}{ccc}
\hline Componentes & $\begin{array}{c}\text { Pan enriquecido con harina de } \\
\text { quinua y soja }(*)\end{array}$ & Pan francés \\
\hline Energía (Kcal) & 295.6 & 291 \\
Humedad $(\mathrm{g})$ & 29 & 27 \\
Proteínas $(\mathrm{g})$ & 12.4 & 8.4 \\
Grasa $(\mathrm{g})$ & 4.0 & 0.2 \\
Carbohidratos $(\mathrm{g})$ & 52.5 & 63.8 \\
Cenizas $(\mathrm{g})$ & 2.5 & 1.5 \\
Hierro $(\mathrm{mg})$ & 6.2 & 1.0 \\
\hline
\end{tabular}

Fuente: Elaboración en la investigación "Elaboración y valoración del hierro en el pan enriquecido con harina de quinua (Chenopodium quinoa w.) y soja (Glycine $\max )(*) 70 \%$ harina de trigo, $10 \%$ harina de quinua, $20 \%$ harina de soja (según la formulación) Collazos C, White H, Viñas E, Alvistur E, Uriquieta R, Vásquez J et al.

Tablas Peruanas de Composición de Alimentos. Ministerio de Salud, Instituto Nacional de Salud y Centro Nacional de Alimentación y Nutrición. $8 .^{\circ}$ ed. Lima, Perú; 20095.

El pan es un alimento que está presente todos los días la dieta, sin embargo, las variedades que se encuentran hoy en día son muchas, por ello la interrogante en este estudio fue cuál es mejor para la salud. Las diferencias entre los tipos de pan dependen del tipo de harina empleada y del grado de refinamiento de la misma. La harina se hace de semillas de trigo, que tienen tres partes ricas en nutrientes: el salvado (las capas externas), el germen (la zona más interna) y el endospermo (la parte almidón en el medio). La harina blanca, por su parte, utiliza solo el endospermo. Al ser comparados con el pan enriquecido que presenta como ingrediente la quinua que es fuente vegetal de proteínas, no tanto debido a su cantidad sino a su calidad, lo cual le otorga un alto valor biológico. La calidad de las proteínas depende de la composición de los aminoácidos, especialmente de la cantidad de aminoácidos esenciales. Así, la lisina, uno de los aminoácidos esenciales más escasos en los alimentos de origen vegetal, está presente en la quinua en proporciones que prácticamente duplican las existentes en los otros cereales y soja leguminosa el que también se encuentran reunidos todos los aminoácidos esenciales; como se muestra en la tabla 11, donde el pan francés es escasamente nutritivo, mientras que el pan enriquecido presenta contenido superior no solo en hierro, sino también en proteínas.

\section{CONCLUSIONES}

El continuo crecimiento del consumo de harina de trigo no logra abastecer su demanda, lo cual incrementa permanentemente su valor, encareciendo gradualmente la elaboración de los insumos utilizados como materia prima, limitando el desarrollo de la industria de la panificación; por lo tanto, las harinas de otros cereales y leguminosas que se producen en el Perú, como quinua y soja, representa una buena opción en ofrecer panes con valor agregado.

El pan enriquecido con harina de quinua y soja contiene cantidades superiores de hierro, proteínas y aminoácidos, haciéndolo de alto valor nutritivo frente al pan francés.

El análisis proximal del producto final alcanzó $6.2 \mathrm{mg} / 100 \mathrm{~g}$ de hierro marcando una diferencia significativa en el contenido de hierro, con respecto al pan francés que muestra $1 \mathrm{mg} / 100 \mathrm{~g}$ descritas en la Tabla de Composición de Alimentos. De acuerdo a los resultados de medición del grado de aceptabilidad, se determinó que la muestra 1 presentó una mayor aceptación de los jueces con respecto a la muestra 2 . 


\section{REFERENCIAS BIBLIOGRÁFICAS}

Carrasco R. 1998. Cultivos andinos: Importancia nutricional y posibilidades de procesamiento. Perú: Didi de Arteta.

Collazos C, White H, Viñas E, Alvistur E, Uriquieta $\mathrm{R}$, Vásquez $\mathrm{J}$ et al. 2009. Tablas peruanas de composición de alimentos. Ministerio de Salud, Instituto Nacional de Salud y Centro Nacional de Alimentación y Nutrición. $8^{\circ}$ ed. Lima, Perú.

Figueroa L. 2006. El libro de la soja. Buenos Aires: Papel y Pluma.

Food and Agriculture Organization/World Health Organization (FAO/WHO). (2002) Requirements of Vitamin A, Iron, Folate and Vitamin B12.

Freire W. 1998. La anemia por deficiencia de hierro: estrategias de la OPS/OMS para combatirla. Scientific electronic library online.

Kent N. Technology of Cereals: An Introduction for Students of Food Science and Agriculture. $4^{\circ}$ ed. EE.UU: Pergamon Kingdom.

León M, Villacorta M. 2010. Valor nutritivo de pan con sustitución parcial de harina de trigo (Triticum aestivum) por arracacha (Arracacia xanthorrhiza Bancroft), fortificado. Revista Venezolana de Ciencia y Tecnología de Alimentos; 1 (2): 244-261.

Ordóñez G, Oviedo R. 2010. Alternativas de aprovechamiento de harinas no tradicionales para la elaboración de pan artesanal. (Tesis para optar el Título de Ingeniero de Alimentos). Escuela Superior Politécnica del Litoral. Guayaquil, Ecuador. 